\title{
Categorização dos pontos estratégicos da fisiologia de voo para o transporte aeromédico
}

RESUMO | Objetivo: Categorizar os pontos estratégicos da fisiologia de voo que possam interferir no transporte aeromédico. Método: Trata-se de um estudo de revisão integrativa de literatura, realizada com base no modelo PRISMA - Preferred Reporting Items for Systematic Reviews and Meta-Analyses. A busca dos artigos foi realizada nos meses de agosto e setembro de 2021. Resultado: Foram utilizados 10 trabalhos, elencadas seis categorias: (i) Altitude; (ii) Áreas comuns que precisam de atenção; (iii) Forças de Aceleração; (iv) Hipóxia, (v) Preparação para o voo do paciente; (vi) Umidade, Temperatura e Gravidade. Conclusão: O transporte em aeronaves de asa fixa necessita de um conhecimento de fisiologia de voo, potenciais alterações na altitude, recomendações específicas, equipe de saúde e tripulação capacitadas para reconhecer e intervir. Assim como, possuam práticas avançadas, compartilhem as informações, maximizem os processos de segurança e qualidade no ambiente hipobárico.

Descritores: Enfermeiros; Fisiologia; Resgate Aéreo; Capacitação; Gestão do Conhecimento.

\begin{abstract}
Objective: To categorize the strategic points of flight physiology that may interfere with aeromedical transport. Method: This is an integrative literature review study, based on the PRISMA model - Preferred Reporting Items for Systematic Reviews and Meta-Analyses. The search for articles was carried out in August and September 2021. Result: 10 works were used, listed in six categories: (i) Altitude; (ii) Common areas that need attention; (iii) Acceleration Forces; (iv) Hypoxia, (v) Preparation for the patient's flight; (vi) Humidity, Temperature and Gravity. Conclusion: Transportation in fixed-wing aircraft requires knowledge of flight physiology, potential changes in altitude, specific recommendations, health care team and crew trained to recognize and intervene. As well as having advanced practices, share information, maximize safety and quality processes in the hypobaric environment.
\end{abstract}

Descriptors: Nurses; Physiology; Air Rescue; Training; Knowledge management.

RESUMEN | Objetivo: Categorizar los puntos estratégicos de la fisiología del vuelo que pueden interferir con el transporte aeromédico. Método: Este es un estudio de revisión de literatura integradora, basado en el modelo PRISMA - Ítems de reporte preferidos para revisiones sistemáticas y metaanálisis. La búsqueda de artículos se realizó en los meses de agosto y septiembre de 2021. Resultado: se utilizaron 10 obras, clasificadas en seis categorías: (i) Altitud; (ii) Áreas comunes que necesitan atención; (iii) Fuerzas de Aceleración; (iv) Hipoxia, (v) Preparación para el vuelo del paciente; (vi) Humedad, temperatura y gravedad. Conclusión: El transporte en aeronaves requiere conocimientos de fisiología de vuelo, posibles cambios de altitud, recomendaciones específicas, equipo de atención médica y tripulación capacitados para reconocer e intervenir. Además de contar con prácticas avanzadas, compartir información, maximizar los procesos de seguridad y calidad en el ambiente hipobárico Descriptores: Enfermeras; Fisiología; Rescate aéreo; Capacitación; Conocimiento administrativo.

\section{Bruno Gonçalves da Silva}

Enfermeiro (UNIFENAS), Mestre em Administração de Empresas (Centro Universitário UNA), Doutorando em Sistemas de Informações e Gestão do Conhecimento (Universidade FUMEC). Professor Assistente FCMMG e Enfermeiro de Bordo da Unimed Aeromédica. ORCID: 0000-0001-5173-0036

\section{Vânia Paula de Carvalho \\ Enfermeira (PUC-MINAS), Enfermeira Intensi- vista (IEC-PUC MG) e Enfermeira Aeroespa- cial (FIC-UNIVIRTUAL), Mestre em Promoção da Saúde e Prevenção da Violência (UFMG). Coordenadora de Enfermagem/RT da Uni- med Aeromédica. \\ ORCID: 0000-0002-9336-3606}

\section{Maria Eduarda Becho Arger Marchetti}

Médica (UFMG), Especialista em Anestesiologista e Medicina Aeroespacial. Médica Emergencista e Horizontal na UPA-CS BH, Anestesista em HCMG e Médica de Bordo na Unimed Aeromédica.

\section{ORCID: 0000-0002-0242-243X}

\section{André Alves Elias}

Médico (UNIFENAS). Médico de Bordo na

Unimed Aeromédica e BOA-SAMU BH.

ORCID:0000-0002- 1103-6450

\section{Flávio Lopes Ferreira}

Médico (UFMG), Mestre em Fisiologia e Farmacologia (UFMG), Cirurgião Geral e Especialista em Medicina Aeroespacial. Gestor Médico da Unimed Aeromédica, Professor na Faculdade de Ciências Médicas de Minas Gerais.

ORCID: 0000-0001-7740-4394

\section{Armando Sérgio de Aguiar Filho}

Comunicador Social, Professor Titular na Universidade FUMEC e da Faculdade Promove. Doutor em Gestão da Informação e do Conhecimento (UFMG).

ORCID: 0000-0001-5542-7165
Recebido em: 22/09/2021

Aprovado em: 08/10/2021

INTRODUÇÃO

A s origens do transporte médico aéreo datam do final do século XIX, época em que balões de ar quente foram utilizados para tirar os feridos do campo de batalha1 e o primeiro relato de transporte aéreo bem-sucedido aconteceu no Cerco de Paris por alemães, em 1870, com o uso de balões de ar quente2, mas este método de transporte só alcançou sucesso noventa anos depois. A lista de balões e aparelhos aéreos que Santos Dumont projetou ou construiu tem lacunas, inconsistências e dados implausíveis que não se explicam. 3

A primeira metade do século $X X$ 
assistiu a um rápido desenvolvimento das capacidades de transporte médico aéreo. Mantendo os avanços na ciência militar, a Força Aérea dos EUA comandou uma abordagem de equipe para o transporte aéreo de cuidados críticos em 1994 na 59a Ala Médica da Base Aérea de Lackland, chamando-a de 'equipe de transporte aéreo de cuidados críticos. 4

Neste sentido, o transporte médico aéreo civil é cada vez mais utilizado, cabe aprender com experiências anteriores, tanto militares quanto civis, com o intuito de melhorar os processos, o atendimento aos pacientes críticos e os resultados, segurança e qualidade.

Para tal, a partir da práxis cotidiana surgiu a discussão com um grupo de pesquisadores especialistas em transporte aéreo de pacientes criticamente enfermos. Seguiu-se a pergunta norteadora: "quais os tópicos a serem estudados sobre fisiologia de voo prévio a um atendimento aeromédico?" Assim, o objetivo deste estudo foi o de categorizar os pontos estratégicos da fisiologia de voo que possam interferir no transporte aeromédico.

\section{MÉTODO}

Trata-se de um estudo de revisão integrativa de literatura, realizada com base no modelo PRISMA - Preferred Reporting Items for Systematic Reviews and Meta-Analyses.5

A busca dos artigos foi realizada nos meses de agosto e setembro de 2021, nos portais online: Periódicos da Capes, Biblioteca Virtual de Saúde (BVS) e Scientific Electronic Library Online (SCIELO). Foram utilizadas as palavras-chaves: "gestão do conhecimento", "transporte de pacientes", "fisiologia de voo" e os seus respectivos em língua inglesa. Para a seleção dos artigos foram utilizados os seguintes critérios: estudos publicados entre 2006 e 2021 que abordassem o processo de formação dos profissionais do serviço aero-

\section{Mantendo os} avanços na ciência militar, a Força Aérea dos EUA comandou uma abordagem de equipe para o transporte aéreo de cuidados críticos em 1994 na 59 Ala Médica da Base Aérea de Lackland, chamando-a de 'equipe de transporte aéreo de cuidados críticos. médico. Foram excluídos trabalhos não encontrados na íntegra, publicações anteriores a 2006 e em idiomas que não fossem o inglês e o português.

A seleção teve início pela identificação das publicações a partir dos seus títulos, seguida pela leitura dos resumos e incluídos aqueles que tivessem todos os critérios após a leitura na íntegra, conforme descrição da figura 1.

Foram localizadas o total de 307 publicações, cinco trabalhos estavam em duplicidade ao realizar as análises dos resultados. Na sequência, fez-se uma seleção dos trabalhos que contemplavam o assunto por leitura de título e resumo, chegando a 22 artigos, ao realizar a leitura na íntegra foram excluídos 12 e incluídos 10 trabalhos.

\section{RESULTADOS}

Os 10 estudos selecionados e descritos foram publicados em periódicos internacionais, conforme o Quadro 1.

\section{DISCUSSÃo}

Os sistemas de transporte aeromédico podem incluir aeronaves de asa rotativa (helicóptero) ou de asa fixa (avião). Além disso, a tripulação de saúde pode ser responsável pelo transporte terrestre (ambulância) de e para o hospital, aeroporto ou heliponto. Para tal, a equipe deve estar familiarizada com a segurança e ser capaz de atuar nos diferentes tipos de ambulâncias em seu sistema. Análogo aos sistemas de emergência (Emergency Medical Services EMS), a tripulação aeromédica opera, utiliza os protocolos e as ordens permanentes, indiretamente. No Brasil, pode ser qualquer combinação dos seguintes profissionais: médico/a; enfermeiro/a e o perfusionista.2

Desta forma, o conhecimento da medicina aeroespacial e da física são informações vitais para as equipes no transporte médico aéreo. As chaves para uma evacuação aeromédica 
bem-sucedida são o planejamento, a resposta a qualquer deterioração na condição que determinou o transporte urgente e as condições induzidas pelo ambiente aeroespacial. 11 Vale ressaltar que, a categorização dos seis pontos estratégicos está relacionada à fisiologia de voo e, consequentemente alinhada a fisiopatologia do paciente criticamente enfermo, no ambiente aéreo.

\section{Altitude}

No que diz respeito à fisiologia aeroespacial, existem diferentes zonas fisiológicas da atmosfera que se tornam relevantes. A zona eficiente é definida como uma altitude de 10.000 pés $(3.000 \mathrm{~m})$ acima do nível do mar. Nesta zona, os níveis de oxigênio são tipicamente adequados para manter a fisiologia da linha de base sem forte necessidade de oxigênio suplementar ou equipamento. No entanto, ao atingir a zona deficiente da atmosfera, 10.000 pés a 50.000 pés acima do nível do mar, haverá uma diminuição na pressão barométrica, bem como diminuirá a pressão parcial do oxigênio. 8

$\mathrm{Na}$ zona deficiente, a maioria dos voos operam e é, também, onde os eventos fisiológicos ocorrem durante o transporte aeromédico. Para a melhor compreensão dos eventos que ocorrem durante os voos devem ser dadas a atenção às leis de Boyle e de Dalton. 8

Áreas comuns que precisam de atenção

Os pesquisadores australianos afirmam que, a comunicação efetivada de maneira direta entre médicos do transporte aeromédico e médicos do hospital de destino podem melhorar a exatidão das informações clínicas para o bem-estar do paciente.10

Durante o diálogo entre as equipes, as informações mínimas devem ser retransmitidas. Existem ferramentas padronizadas que garantem a melhor comunicação entre as equipes, ou seja, o uso de checklists e a retransmissão de informações de forma estruturada. 8
Figura 1. Fluxograma de seleção dos estudos, de acordo com os critérios de inclusão e exclusão pré-estabelecidos.

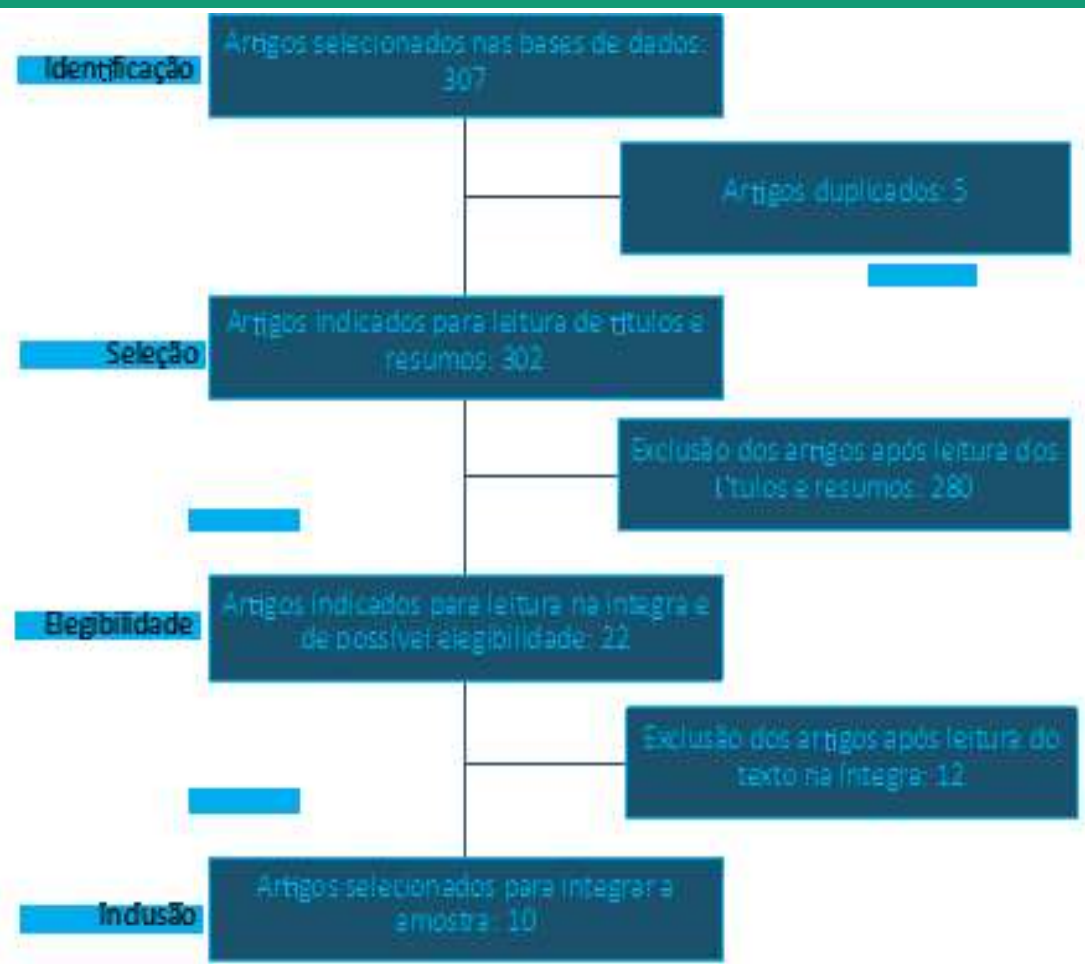

Fonte: Elaborado pelos autores, 2021.

Quadro 1. Títulos dos estudos, as categorias abordadas e os anos que foram publicados

$\begin{array}{ccc}\text { Títulos } & \text { Anos } & \text { Categorias } \\ \begin{array}{c}\text { Preparation of the critical } \\ \text { patient for aeromedical } \\ \text { transport.6 }\end{array} & 2014 & \text { Preparação para o voo do paciente } \\ \text { Áreas comuns que precisam de atenção }\end{array}$

Recommendations for the intra-hospital transport of critically ill patients.7

\begin{tabular}{|c|c|c|}
\hline $\begin{array}{l}\text { Principles of air transport phy- } \\
\text { siology for JMATT clinicians. } 8\end{array}$ & 2012 & Altitude \\
\hline $\begin{array}{l}\text { Aero-medical considerations } \\
\text { in casualty air evacuation } \\
\text { (CASAEVAC). } 9\end{array}$ & 2010 & Preparação para o voo do paciente \\
\hline $\begin{array}{l}\text { iSoBAR - a concept and han- } \\
\text { dover checklist: The national } \\
\text { clinical handover initiative. } 2,9\end{array}$ & 2009 & Áreas comuns que precisam de atenção \\
\hline $\begin{array}{l}\text { Critical Considerations for } \\
\text { Fixed-Wing Air Medical } \\
\text { Transports.2 }\end{array}$ & 2019 & $\begin{array}{c}\text { Hipóxia } \\
\text { Umidade, Temperatura e Gravidade } \\
\text { Forças de Aceleração }\end{array}$ \\
\hline
\end{tabular}




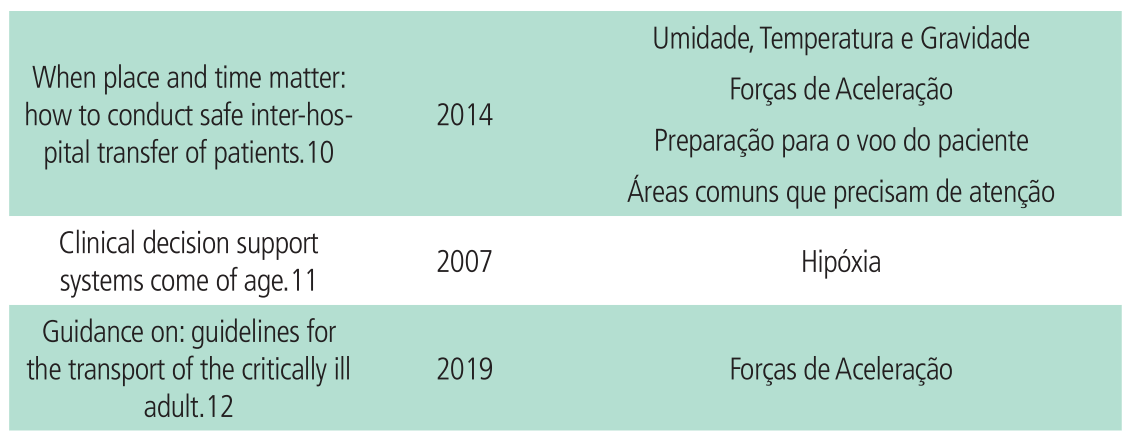

Fonte: Elaborado pelos autores, 2021.

\section{Tabela 1 - Mudanças de oxigênio e temperatura em altitudes²}

\begin{tabular}{cccccc}
$\begin{array}{c}\text { Altitude } \\
\text { (pés) }\end{array}$ & $\begin{array}{c}\text { Pressão } \\
\text { atm/t* } \\
(\mathbf{m m H g})\end{array}$ & $\begin{array}{c}\text { Pa02 } \\
(\mathbf{m m H g})^{*}\end{array}$ & $\begin{array}{c}\text { Pa02 } \\
(\mathbf{m m H g})^{* *}\end{array}$ & $\begin{array}{c}\text { Saturação } \\
\text { de oxigê- } \\
\text { nio (\%) }\end{array}$ & $\begin{array}{c}\text { Temperatura } \\
\text { em graus } \\
\text { Celsius }\end{array}$ \\
$\begin{array}{c}\text { Nivel do } \\
\text { mar }\end{array}$ & 760 & 160 & 100 & 98 & 15 \\
8.000 & 565 & 118 & 69 & 93 & -0.8 \\
10.000 & 523 & 110 & 60 & 87 & -4.8 \\
\hline 18.000 & 379 & 80 & 38 & 72 & -20.7 \\
\hline 20.000 & 349 & 73 & 34 & 66 & -24.6 \\
\hline 35.000 & 179 & 38 & 0 & 0 & -55 \\
\hline
\end{tabular}

Notas: $\left(^{*}\right)$ Atmosférica total; $\left(^{* *}\right)$ Pressão parcial do oxigênio alveolar; $\left(^{* * *}\right)$ Pressão parcial do oxigênio arterial Fonte: Adaptado pelos autores, 2021

Ainda, a ferramenta recomendada para padronizar a comunicação relativa às informações do paciente é o iSoBAR (identify-situation-observations-background-agreed plan-read back), ou seja, identificar a situação e o histórico, avaliar e recomendar. Dito de outra forma, uma boa comunicação sempre favorece os cuidados ao paciente e para melhoria dos resultados.9,10

\section{Forças de Aceleração}

A análise das forças gravitacionais experimentadas em aceleração e desaceleração, podem impactar em alterações dos processos fisiológicos dos pacientes críticos e os efeitos poderão ser exacerbados.10 $\mathrm{Na}$ aceleração podem cursar com hipertensão, disritmias, mudanças nos fluidos compartimentais, taquicardia, aumento da pressão intracraniana.2,10 Ainda, os efeitos do ruído e da vibração em um trans- porte aéreo podem causar dor, náusea e ansiedade e, portanto, devem ser reconhecidos pelas equipes de saúde e devem ser tratados adequadamente. 1

\section{Hipóxia no voo}

No estudo da fisiologia relacionada as altitudes, a consequência mais comum no voo é a hipóxia. A Lei de Dalton ajuda a explicar seu mecanismo, afirmando que a pressão total de uma mistura de gás é igual à soma da pressão parcial de cada gás na mistura: $\mathrm{PT}=\mathrm{P} 1+\mathrm{P} 2++\mathrm{PN}$. Ao aumentar a altitude, a pressão parcial do oxigênio diminui. Para combater esse efeito as aeronaves modernas voam com cabines pressurizadas, com altitude aproximada de 8.000 pés.2 Assim, os pacientes com hipóxia ou comprometimento respiratório na fase pré voo, provavelmente, irão deteriorar o padrão respiratório durante o traslado se não forem feitos esforços para otimizar a sua oxigenação. 6

A lei de Boyle, explica que o volume de um gás é inversamente proporcional à pressão à qual é submetido", $\mathrm{P} 1 / \mathrm{P} 2=\mathrm{V} 1 / \mathrm{V} 2$. Com base nessa lei, a pressão diminui com o aumento da altitude e causa, assim, o aumento no volume do gás, o que leva ao conceito de expansão do gás.

Diante do exposto, consequências potenciais da retenção/expansão dos gazes podem acontecer.11 Existem espaços nas diversas cavidades corporais suscetíveis de expansões durante o voo e, consequentemente, de complicações. Ainda, alguns esforços são necessários para equalizar as pressões das respectivas cavidades e o profissional de saúde deve estar preparado para intervir.

\section{Preparação para o voo do paciente}

Faz-se necessário que, durante o preparo do paciente criticamente enfermo, aerorremovido, em aeronaves asa fixa, haja o devido planejamento e a execução seja impecável. A preparação do paciente deve ser estendida a todos envolvidos nos processos. Pois, a abordagem holística da transferência proporciona melhoria nos resultados e otimiza a segurança. Os processos de transferência de pacientes devem ser pautados na utilização de procedimentos padronizados, pois podem ajudar a mitigar os riscos associados.7

A etapa crítica do transporte ao paciente é a avaliação e a estabilização de suas vias aéreas. Caso não esteja com via aérea definitiva e/ou em uso de ventilação mecânica, a equipe de saúde de bordo deve avaliar, identificar e intervir em casos de comprometimento por desconforto ou insuficiência respiratória. De acordo com a gravidade, os dispositivos ventilatórios serão instituídos e faz-se necessário que ele seja intubado eletivamente para o transporte. 10

O gerenciamento das vias aére- 
as deve ser realizado na fase pré voo, idealmente. Os pacientes com uso de próteses ventilatórias (tubos, máscaras laríngeas e outros) devem utilizar fixadores eficientes (há vários modelos no mercado) e que consigam evitar perdas dos dispositivos.6,13

\section{Umidade, Temperatura e Gravidade}

Dentre os pontos estratégicos, há outras considerações fisiológicas que incluem baixa umidade, mudanças de temperatura e os efeitos das forças gravitacionais. Com relação à baixa umidade da cabine, os pacientes experimentam ressecamento das secreções (olhos, nariz, boca, trato respiratório, outros), desidratação e aumento de muco. 10

Os pesquisadores Pritchard et al. (2019) demostraram, que à medida que a altitude aumenta, a temperatura atmosférica, a pressão parcial de oxigênio e a umidade diminuem. O risco de epistaxe também é aumentado e para isso, a tripulação de saúde necessita estar preparada para gerenciar essa complicação e dar a melhor assistência possível ao paciente. 2 Ao reconhecer o ambiente hostil e o efeito esperado a assistência será organizada e segura,10 conforme demonstra a Tabela 1 .

Neste contexto, as seis categorias e as suas particularidades foram descritas sucintamente. Espera-se que, este estudo tenha contribuído para a meIhoria da compreensão da fisiologia de voo para o transporte de pacientes críticos, com o compartilhamento das informações e a gestão do conhecimento. É sabido que, existem outros fatores estressores, particularidades do ambiente, necessidade de uma equipe multidisciplinar capacitada. Para tal, o ambiente aéreo com equipes altamente especializadas, otimiza a segurança e a qualidade da assistência.14

\section{CONCLUSÃO}

O transporte aéreo de pacientes em aeronaves de asa fixa necessita de um conhecimento de fisiologia de voo, potenciais alterações em altitude e recomendações específicas. Para isso, a equipe de saúde e a tripulação devem ser capacitados para reconhecer e intervir, de acordo com a necessidade.

Diante do exposto, é importante que os profissionais de saúde possuam práticas avançadas, capacitação, compartilhem as informações entre os pares, conheçam a fisiologia de voo, maximizem os processos de segurança e qualidade no ambiente hipobárico.

Assim, o objetivo deste estudo foi o de categorizar os pontos estratégicos da fisiologia de voo que possam interferir no transporte aeromédico. Esse estudo sugeri novas pesquisas para cobrir as lacunas de publicações.

\section{Referências}

1. Fromm Re, Varon J. Critical care transport. Critical Care Clinic. 2000;16(4):695-705.

2. Pritchard J, Di Corpo Je, Torres A, Merlin Ma, Schwarbaum J, et al. Critical Considerations for Fixed-Wing Air Medical Transports. JEMS Journal of Emergency Medical Services. 2019 Mar;1-21.

3. Ramalho V. As biografias históricas de Santos Dumont. Scientiae Studia. 2013;11(3):687-705.

4. Gissom TE, Farmer JC. The provision of sophisticated critical care beyond the hospital: Lessons from physiology and military experiences that apply to civil disaster medical response. Critical Care Medicine. 2005;33(1):13-21.

5. Galvão TF, Pansani T De Sa, Harrad D. Principais itens para relatar Revisões sistemáticas e Meta-análises: A recomendação PRISMA. Epidemiologia e Serviços de Saúde. 2015; 24:335-42.

6. Le Cong M, Ramin G. Preparation of the critical patient for aeromedical transport. Prehospital and Retrieval Medicine. 2014 Jun.

7. Fanara B, Manzon C, Barbot 0 , et al. Recommendations for the intra-hospital transport of critically ill patients. Critical Care Medicine. 2010;14(3):87.

8. Fowler JM. Principles of air transport physiology for JMATT clinicians. Critical
Care Air Transport. 2012 Jan.

9. Joshi MC, Sharma RM. Aero-medical considerations in casualty air evacuation (CASAEVAC). Medical Journal of Armed Forces India. 2010;66(1).

10. Porteous JM, Stewart-Wynne EG, Connolly M, et al. iSoBAR - a concept and handover checklist: The national clinical handover initiative. Medical Journal of Australia. 2009;190(11):152-6.

11. Sethi $D$, Subramanian $S$. When place and time matter: how to conduct safe inter-hospital transfer of patients. Saudi Journal of Anaesthesia. 2014;8(1):104-13.

12. Teich JM, Wrinn MM. Clinical decision support systems come of age. MD Computing. 2007;17(1):43-6.

13. Tillbrook-Evans D. Guidance on: guidelines for the transport of the critically ill adult. 3rd ed. London: FICM Faculty of Intensive Care Medicine. 2019;

14. da Silva, B. G., Viana, L. L., Faustino, S. D. S. F., Silveira, C. D. P. S., de CarvaIho, V. P., \& de Aguiar Filho, A. S. (2021). Preparação do enfermeiro para o atendimento à múltiplas vítimas no resgate aéreo. Nursing (São Paulo), 24(278), 5948-5957. 